\title{
Parabacteroides goldsteinii
}

National Cancer Institute

\section{Source}

National Cancer Institute. Parabacteroides goldsteinii. NCI Thesaurus. Code C123535.

A species of oblig ately anaerobic, non-spore-forming, non-motile, Gram negative, rodshaped bacterium in the family Porphyromonadaceae. This species is negative for urease and nitrate reduction. Acid is produced from cellobiose, glucose, rhamnose, sucrose, trehalose and xylose, but not from arabinose or xylan. P. goldsteinii was isolated from human clinical specimens of intestinal origin. 